\title{
CAMBIOS FÍSICO-QUÍMICOS DEL AGUACATE (Persea americana Mill. cv. "Hass") EN POSCOSECHA PARA DOS MUNICIPIOS DE ANTIOQUIA
}

\section{CHANGES PHYSICAL-CHEMICAL OF AVOCADO (Persea americana Mill. cv. "Hass") IN POSTHARVEST FOR TWO MUNICIPALITIES OF ANTIOQUIA}

\author{
Carlos J. Márquez ${ }^{1 *}$, IA, MSc, Dr. Sc., Diana P. Yepes², Ing. de Alimentos, MSc, Laura Sanchez³ , Ing. \\ Quimica, Jairo A. Osorio ${ }^{4}$, MSc, Dr. Sc.
}

Recibido para publicación: Febrero 21 de 2014 - Aceptado para publicación: Mayo 23 de 2014

\begin{abstract}
RESUMEN
Las características fisiológicas y físico químicas de los aguacates (Persea americana Mill. cv. Hass) cultivados en los municipios del Carmen de Viboral y El Retiro ubicados en el Departamento de Antioquia (Colombia) son desconocidas, es por ello que el objetivo de esta investigación fue caracterizar los frutos provenientes de dichas regiones, los cuales fueron almacenados durante 21 días a $23^{\circ} \mathrm{C}$ y $65 \%$ de HR en promedio. Dentro de los parámetros fisiológicos evaluados se determinaron: tasa de respiración y pérdida fisiológica de peso, también se valoraron las características fisicoquímicas, como el color a través de la medida de los parámetros triestimulos $\mathrm{L}^{*}, \mathrm{a}^{*}, \mathrm{y} \mathrm{b}^{*}$, firmeza, rendimiento en pulpa del fruto, contenido de humedad, materia seca, sólidos solubles totales (SST), acidez total titulable, $\mathrm{pH}$ y contenido lipídico. Se pudo comprobar que la tasa de respiración de los frutos de aguacate es típica de productos climatéricos monofásicos, presentando el pico climatérico en el día 8 de poscosecha, lo que probablemente es la causa de la cascada de eventos propios de la maduración, donde sobresalen los cambios en los SST los cuales fueron de 2,45 para el día 14 de poscosecha, la acidez total titulable disminuyó entre los días 1 a 10 de 0,25 a 0,18\% y la firmeza presentó un decrecimiento continuo pasando de 67 Newton para el día 1 de poscosecha hasta 7,7 para el día 14 de poscosecha, además se pudo establecer una concentración de la fracción lipídica del 24\% y del 22,5\% para los aguacates cosechados en el Carmen de Viboral y El Retiro.
\end{abstract}

Palabras clave: Respiración, características fisicoquímicas, parámetros fisiológicos.

\begin{abstract}
Physiological and physico chemical characteristics of avocados (Persea americana Mill. Cv. "Hass") grown in the municipalities of Carmen de Viboral and El Retiro located in Antioquia (Colombia) are unknown, that is why the objective of this research was to characterize the properties for fruits from this regions, the avocados

${ }^{1}$ Ingeniero Agrícola, Doctor en Ciencias Agrarias, Universidad Nacional de Colombia, Sede Medellín. *Autor para correspondencia: Calle 59a No 63-20. Medellín Colombia. Correo electrónico: cjmarque@unal.edu.co

${ }^{2}$ Ingeniera de Alimentos, Magíster en Ciencia y Tecnología de Alimentos, SENA, Área de Agroindustria

${ }^{3}$ Ingeniera Química, Universidad Nacional de Colombia, Sede Medellín

${ }^{4}$ Ingeniero Agrícola, Doctor en Ciencias, Universidad Nacional de Colombia, Sede Medellín
\end{abstract}


were stored for 21 days at $23^{\circ} \mathrm{C}$ and $65 \% \mathrm{RH}$. The physiological parameters evaluated were: the respiration rate and physiological weight loss, physicochemical characteristics were also evaluated as changes in color $L^{*}$, a* and $b^{*}$, firmness, yield pulp fruit, moisture content, dry matter, total soluble solids (TSS), Total titratable acidity, $\mathrm{pH}$, and lipid content. It could be established that the respiration rate of avocado fruits is typical of monophasic climateric products, presenting a climacteric peak for the day 8 postharvest, this probably is the cause of the cascade of events characteristic of the ripening, with changes in the TSS, which were 2.45 for the postharvest day 14 , the total acidity decreased between days 1 to 10 from 0.25 to $0.18 \%$, and the firmness presented a continuous decrease from 67 Newton for day 1 to 7.7 for day 14 in postharvest, also it could be established a concentration of the lipid fraction of $24 \%$ and $22.5 \%$ for avocados harvested in the Carmen de Viboral and El Retiro.

Key words: Respiration, physicochemical characteristics, physiological parameters.

\section{INTRODUCCIÓN}

El aguacate (Persea americana Mill. cv. "Hass") se produce principalmente en México, Indonesia, Estados Unidos, Chile, Colombia y Guatemala (Velásquez 2008; Gutiérrez 2009). Colombia registra en la actualidad el cuarto lugar en producción de este fruto con 27.555 ha sembradas, con rendimiento de 9,3 t/ha, para una producción de 256.261 t/año, Antioquia representa el segundo Departamento productor del país con 4.083 ha sembradas (Agronet 2012).

Los municipios de Antioquia seleccionados para esta investigación son importantes productores de aguacate $y$ a estos frutos no se han hecho estudios en la calidad de poscosecha, aspecto que es de gran interés para esta cadena agroindustrial. Debido a que de ello depende su comercialización, además del adecuado uso para las transformaciones agroindustriales y el uso de los subproductos, especialmente el aceite.

El fruto de aguacate es climatérico, considerado tropical, con características sensoriales excelsas, el cual por ser un fruto de alto contenido lipídico, con ácidos grasos insaturados de relevancia nutraceútica, le brinda potencial para su consumo en fresco o transformado (Devia 2009). La maduración en este vegetal constituye un proceso complejo en el cual hay degradación y construcción de compuestos, lo cual hace que sea de gran relevancia el estudio de la evolución de estas características (Wu et al. 2005; Moreno et al. 2007; Tejacal et al. 2007).

Se estima que a nivel mundial las pérdidas poscosecha de frutas son del $2 \%$ al $23 \%$ en países desarrollados y hasta de $50 \%$ en países en desarrollo (Kader 2005), donde gran parte de estas pérdidas se dan en los procesos de poscosecha, especialmente en manipulación y transporte (Agustí 2004). En aguacate, las pérdidas poscosecha pueden llegar hasta el 30\% por prácticas inadecuadas y desconocimiento del comportamiento del fruto luego de su recolección (Ramírez 2008). Debido a los requerimientos de calidad de los frutos, es pertinente evaluar su comportamiento en poscosecha, particularmente las características fisiológicas y fisicoquímicas durante la etapa de posrrecolección.

El objetivo de ésta investigación fue la determinación de las características fisiológicas 
y fisicoquímicas más relevantes que se relacionan con los cambios ocurridos durante la maduración o periodo poscosecha del aguacate (Persea americana Mill. cv. "Hass"), para frutos cultivados en los municipios del Carmen de Viboral y El Retiro, región del oriente antioqueño.

\section{MATERIALES Y MÉTODOS}

El material vegetal utilizado para la investigación fueron frutos de aguacate cv. "Hass" obtenidos de 4 fincas productoras del Oriente de Antioquia (Colombia), ubicadas 2 por cada municipio, correspondientes a El Carmen de Viboral, vereda El Cerro, localizada a $2.150 \mathrm{msnm}$ con temperatura media de $17^{\circ} \mathrm{C}$ y de El Retiro, veredas Pantanillo y Don Diego, ubicadas a $2.175 \mathrm{msnm}$ con temperatura media de $16^{\circ} \mathrm{C}$. Cada municipio se constituye en un factor dentro del diseño del experimento, al igual que los días de poscosecha y las dos fincas de cada municipio constituyen las réplicas del experimento. Los frutos fueron cosechados en cada finca de 14 árboles, tomando 3 unidades experimentales por árbol, en grado de madurez fisiológica, de acuerdo a las especificaciones de la Norma Técnica Colombiana NTC 5209, correspondientes a frutos ovales piriformes, con cáscara rugosa que de verde se torna morada y luego negra durante el proceso de maduración.

Este material vegetal fue cosechado y de inmediato transportado en canastillas plásticas de $10 \mathrm{~kg}$ hasta la Universidad Nacional de Colombia, Sede Medellín, a los Laboratorios de Frutas y Hortalizas, y de Control de Calidad de Alimentos, donde se realizaron los respectivos análisis.
El experimento se estableció de acuerdo a un diseño completamente al azar, con múltiples variables y respuestas. El trabajo se desarrolló con frutos colectados en una misma época de cosecha correspondiente al mes de Julio de 2010. Para las evaluaciones físico-químicas se dispuso de 42 frutos por finca por 2 fincas por municipio por 2 municipios, para un total de 168 Unidades Experimentales (U.E.) y se analizaron 6 U.E. por día por finca por municipio durante los días 1, 3, 7, 10, 14, 17 y 21 de poscosecha.

La respiración fue analizada con otras 6 U.E. independientes, durante los días 0 a 20, cada 2 días.

Las determinaciones fisiológicas fueron, tasa de respiración (Adaptado de Herridge y Pate 1977; Angueira et al. 2003) y la pérdida fisiológica de peso. La valoración de las características fisico-químicas fueron, color externo o de la epidermis e interno o del mesocarpio (Almela et al. 2000), firmeza (Manenoi 2007), rendimiento en pulpa (Camacho y Romero 1996), contenido de humedad (A.O.A.C. 1984), materia seca, sólidos solubles totales (SST), acidez total, $\mathrm{pH}$ (Bernal de Ramírez 1993) y contenido lipídico de la pulpa (A.O.A.C. 1990).

Los resultados se graficaron con respecto a los días de poscosecha y se aplicaron los procedimientos estadísticos error estándar, coeficiente de variación y promedios aritméticos y análisis de correlación entre alguna variables de interés. 


\section{RESULTADOS Y DISCUSIÓN}

\section{Tasa de respiración y pérdida fisiológica de peso}

La figura 1A presenta el comportamiento de la respiración expresada en $\mathrm{mg}$ de $\mathrm{CO}_{2} \bullet \mathrm{kg}^{-1}$ - $\mathrm{h}^{-1}$ y la $1 \mathrm{~B}$ presenta la pérdida fisiológica de peso expresada en porcentaje (\%) con respecto al peso inicial, para los frutos de aguacate almacenados a $23^{\circ} \mathrm{C}$ y $65 \%$ de HR.

De acuerdo a la figura 1A, la tasa de respiración de los frutos de aguacate mostró un primer periodo constante hasta el día 2 y luego presentó la mayor aceleración en la producción de $\mathrm{CO}_{2}$, con un comportamiento continuo y creciente hasta el día 8, pasando de $20 \mathrm{mg}$ de $\mathrm{CO}_{2} \bullet \mathrm{kg}^{-1} \bullet \mathrm{h}^{-1}$ hasta un valor máximo equivalente a $151,5 \mathrm{mg}$ de $\mathrm{CO}_{2} \bullet \mathrm{kg}^{-1} \bullet \mathrm{h}^{-1}$, siendo éste el pico climatérico, luego se presentó un descenso hasta el día 18 y en las frutas sobre maduras se encontró un ligero incremento respiratorio. Este comportamiento coincide con lo reportado por Eaks (1978) y Villa et al. (2010). La tasa de respiración de los frutos los tipifica como climatéricos monofásicos, lo cual está acorde con lo reportado por Award y Young (1979; Wills (1999) y Márquez
(2009). Los picos respiratorios climatéricos son el reflejo de un incremento en la actividad mitocondrial, debido a la mayor disponibilidad de carboxilatos como sustrato, probablemente inducida por el desacomodo ocasionado por el desprendimiento del árbol, combinado con la degradación delalmidón, poracción enzimática y la formación de carbohidratos de bajo peso molecular, con una actividad específica de tejidos y estructuras muy alta (Bruinsma y Paull 1984). De acuerdo a la producción de $\mathrm{CO}_{2}$, el fruto de aguacate presenta una tasa de respiración extremadamente alta (mayor a 60 mg de $\mathrm{CO}_{2} \bullet \mathrm{kg}^{-1} \bullet \mathrm{h}^{-1}$ ), aspecto que se refleja en lo perecedero del vegetal (Kader 2002).

En cuanto a la pérdida fisiológica de peso, figura $1 \mathrm{~B}$, los frutos de aguacate presentaron un comportamiento continuo creciente durante toda la etapa de poscosecha, con un ligero descenso en la velocidad a partir del día 10. Durante toda la etapa de poscosecha, incluyendo el período de sobremaduración y senescencia, los aguacates tuvieron una pérdida total de peso de $23 \%$ y 22,4\% para los municipios de El Carmen de Viboral y de El Retiro respectivamente.
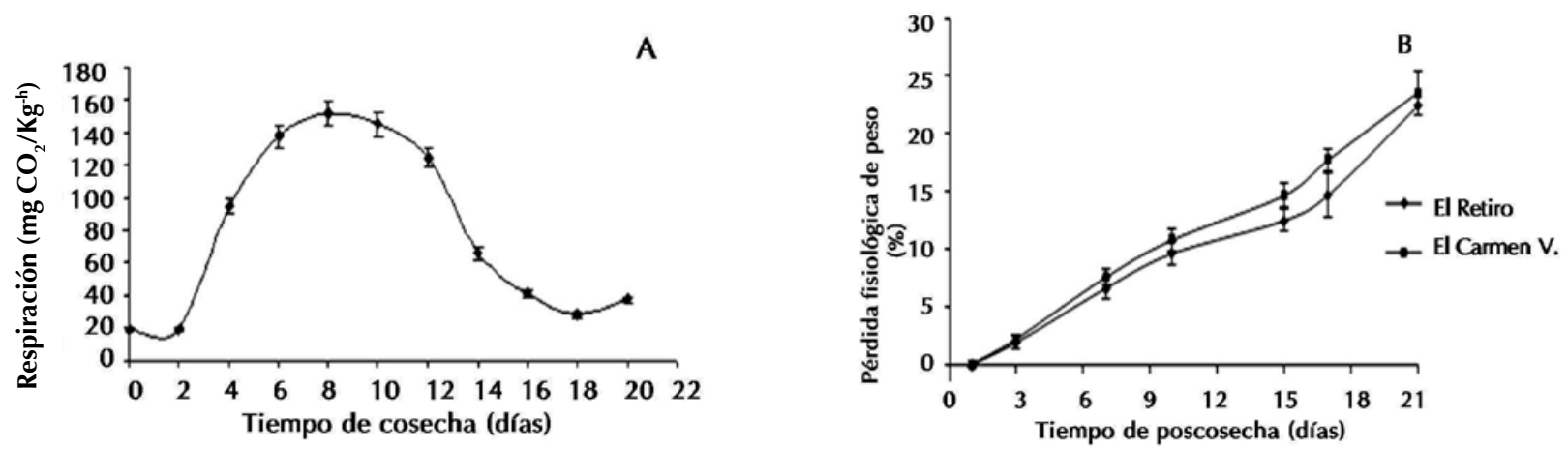

Figura 1. (A). Comportamiento respiratorio para frutos de aguacate (Persea americana Mill cv. Hass) de dos municipios de Antioquia Colombia, y (B). Pérdida fisiológica de peso expresada en porcentaje (\%) con respecto al peso inicial. Los símbolos muestran la media y las barras verticales los valores del error estándar, evaluados durante 21 días poscosecha. 
Los valores encontrados son mayores que los reportados por Lallum et al. (2004) quienes hallaron para frutos almacenados a $20^{\circ} \mathrm{C}$ y 95\% de humedad relativa, pérdidas fisiológicas de peso de $6,7 \%$ en el día 12 de poscosecha. Otros investigadores han reportado una pérdida máxima de peso del $11 \%$ en frutos de aguacate almacenados durante 12 días a $20^{\circ} \mathrm{C}$, lo anterior probablemente debido a la temperatura más baja y humedades relativas más altas para el almacenamiento de los frutos (Cajuste et al.1994).

\section{Color de la epidermis y mesocarpio}

La figura 2 presenta los resultados encontrados para los valores triestimulos del color $L^{*}, a^{*} y$ $b^{*}$, para la epidermis y el mesocarpio de los frutos de aguacate.

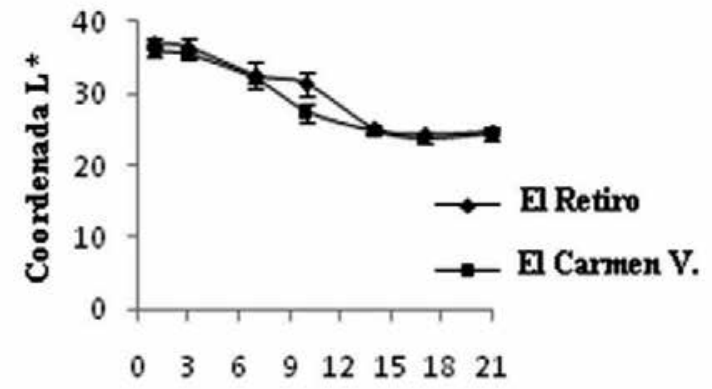

Tiempo de poscosecha (Dias) A
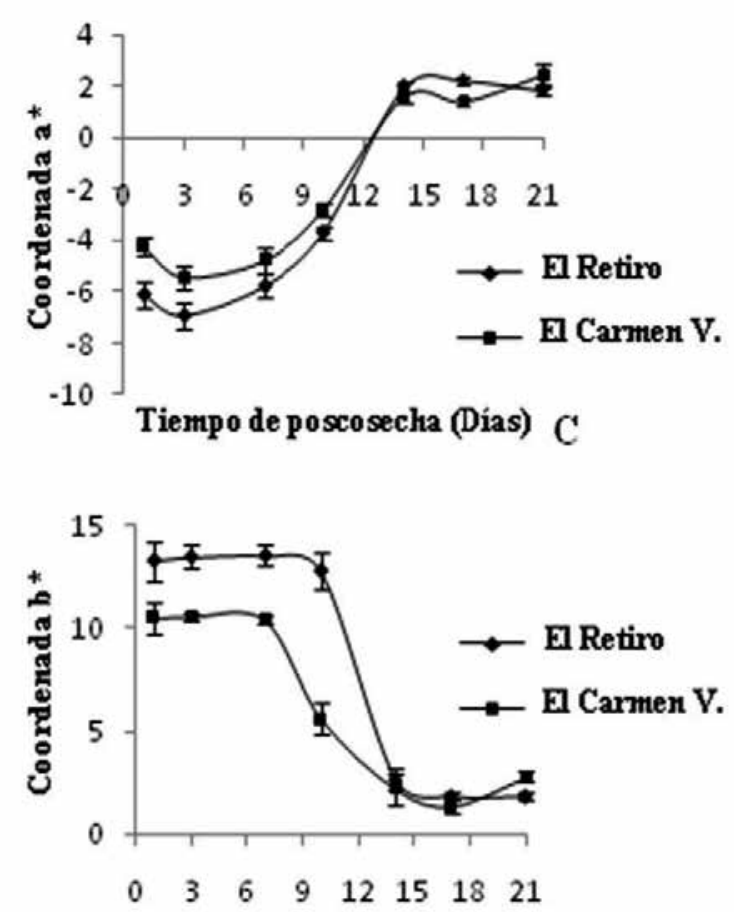

Tiempo de poscosecha (Días) E

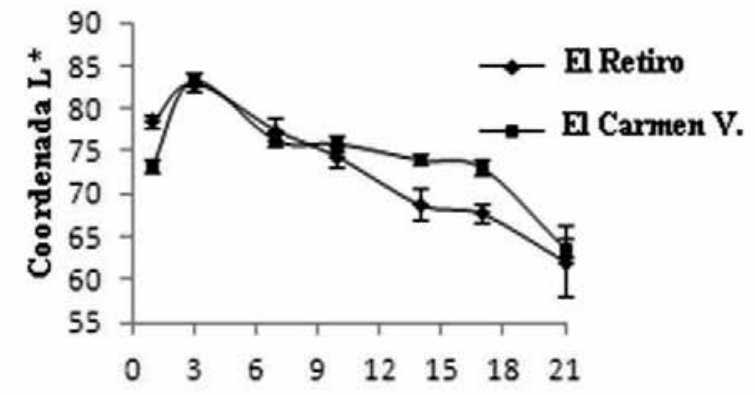

Tiempo de poscosecha (Días) B
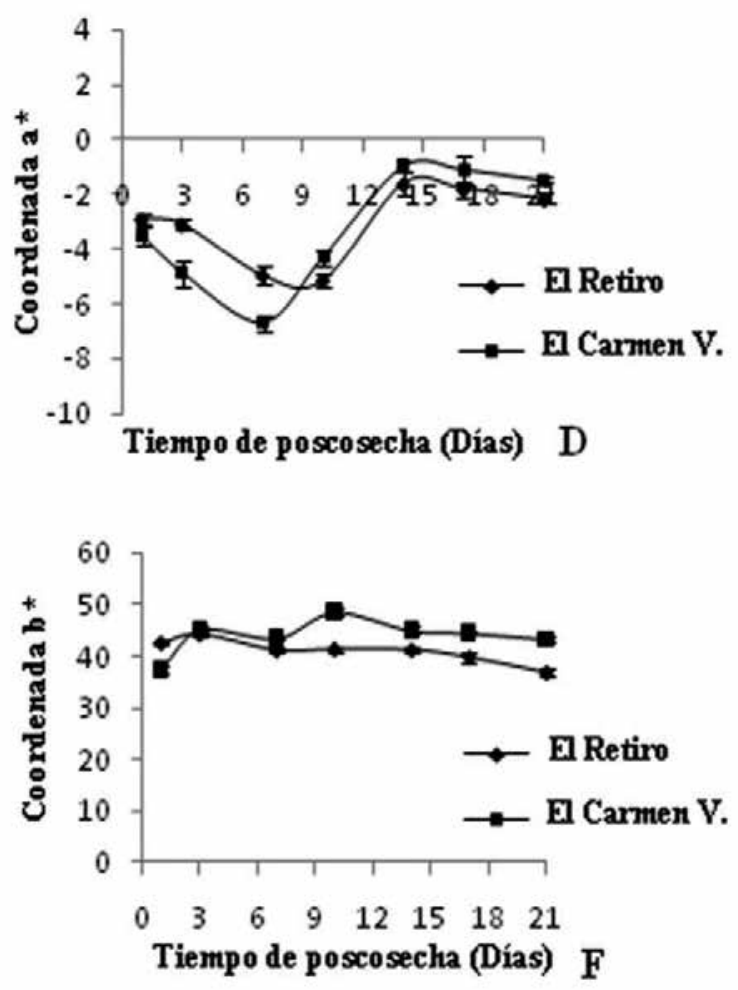

Figura 2. Valores de las coordenadas del color para la epidermis o piel $L^{*}(A), a^{*}(C)$ y $b^{*}(E)$, y para el mesocarpio o pulpa $L^{*}(B), a^{*}(D)$ y $b^{*}(F)$ en frutos de aguacate (Persea americana Mill. cv. Hass) de dos municipios de Antioquia Colombia. Los símbolos representan la media y las barras verticales los valores \pm del error estándar, evaluados durante 21 días de poscosecha. 
En esta figura puede apreciarse como la luminosidad $\left(L^{*}\right)$ en la epidermis de los frutos de aguacate de El Carmen de Viboral y El Retiro presentaron un comportamiento decreciente hasta el día 7 de poscosecha, a partir del cual se encontró un notable aceleramiento en la pérdida de luminosidad y se hizo más intenso entre los días 10 y 14, período que coincide con la madurez de consumo, siendo los frutos del municipio de El Carmen de Viboral los que mostraron mayor disminución de la luminosidad $\left(\mathrm{L}^{*}\right)$.

Para el período de sobre-madurez correspondiente a los días 17 a 21 de poscosecha no se presentaron cambios significativos de la luminosidad $\left(L^{*}\right)$ en la epidermis de los frutos de ambos municipios. En el mesocarpio la luminosidad decreció continuamente durante toda la etapa de poscosecha, siendo más acelerada durante los días 17 a 21, correspondiente a la etapa de sobre-madurez, esta tendencia es similar para los frutos de aguacate de ambos municipios. Los resultados encontrados para la luminosidad, están acordes con los reportados por Cox et al. (2004).

La coordenada a* en la epidermis de los frutos de aguacate de ambos municipios presentó los valores más negativos para los frutos inmaduros (días 1 a 3 de poscosecha) lo cual corresponde a una mayor intensidad en la tonalidad verde, luego mostró un crecimiento que se intensificó en la etapa de madurez de consumo (días 10 a 14 de poscosecha) mostrando valores positivos. Esto quiere decir que hay disminución de la tonalidad verde, probablemente asociado con la degradación de la clorofila b, por acción enzimática, hidrolizándose a clorofilida y fitol (Kader 1991; Yang et al. 2009), presentando el color oscuro característico de los frutos de aguacate cv. Hass.

En la etapa de sobremadurez (días 17 a 21) no se presentaron cambios significativos en la coordenada a*. Para el mesocarpio de los frutos de aguacate de ambos municipios se encontró un comportamiento decreciente (días 1 a 7) el cual se incrementó hasta el día 14, mostrando valores negativos por la contribución del color verde en la pulpa, aspecto que está acorde con lo determinado por Parra et al. (2005).

La coordenada $b^{*}$ en la epidermis de los frutos de ambos municipios no presentó diferencias significativas en la primera etapa poscosecha (día 1 a 7), a partir del cual decreció (día 10 a 14). Lo anterior podría estar asociado con la disminución de clorofila a, debido a que la clorofila $b$ posee una estructura similar a la de la clorofila a, pero el grupo 3-metilo se halla sustituido por el grupo 3-formilo, esta pequeña diferencia, produce cambios en las absorciones de luz visible, y a simple vista el color de una disolución de clorofila b es verde, a diferencia de una de clorofila a que es azul. Por lo tanto, la coordenada de color b*, estaría asociada a la clorofila a, y al reducir su concentración con la maduración igualmente ocasiona una disminución de la coordenada $b^{*}$.

El mesocarpio mostró un comportamiento ligeramente creciente para la coordenada $b^{*}$ hasta el día 10 y luego permaneció constante lo cual podría estar asociado a la síntesis y permanencia de pigmentos carotenoides (Islam et al. 1996). 


\section{Firmeza}

En la figura 3 se puede apreciar como los frutos de aguacate de ambos municipios durante la poscosecha presentaron disminución en su firmeza. En el día 1 se presentó la mayor resistencia a la penetración con valores de firmeza de 67 y 60 Newtons (N) para los frutos del municipio de El Carmen de Viboral y de El Retiro.

La mayor aceleración en el descenso de la firmeza se dio entre el día 10 a 14, período en el cual el valor decreció de $52 \mathrm{~N}$ a $10 \mathrm{~N}$ para los frutos de El Carmen de Viboral y de $50 \mathrm{~N}$ a 8 $\mathrm{N}$ para los frutos de El Retiro, lo cual es similar a lo reportado por Correa et al. (1995) quienes encontraron una disminución en la firmeza de $76,5 \mathrm{~N}$ a 3,1 $\mathrm{N}$ en frutos de aguacate $\mathrm{cv}$. "Hass" almacenados a $20^{\circ} \mathrm{C}$ durante 11 días; y por Zauberman y Jobin-Décor (1995) quienes hallaron una disminución en la firmeza de $60 \mathrm{~N}$ a $5 \mathrm{~N}$ en frutos de aguacate cv. "Hass" almacenados a $22^{\circ} \mathrm{C}$ durante 14 días.
En la etapa de sobremadurez (días 17 a 21) la fuerza de firmeza no presentó cambios significativos para los frutos de ambos municipios. Una vez que se alcanzó la madurez fisiológica, la pérdida de firmeza de los frutos de aguacate se tornó progresivamente menor conforme se acercó a la madurez de consumo.

El ablandamiento es el principal aspecto del proceso de maduración en los frutos de aguacate, como consecuencia de modificaciones en la composición y estructura de la pared celular. Los cambios ocurridos son debidos, probablemente a hidrólisis de los compuestos pécticos presentes en la pared celular, por la acción de enzimas pectinasas, poligalacturonasas, celulasas y amilasas, asociados a la pérdida de turgencia celular debida a la transpiración, dando como resultado final el ablandamiento de los frutos de aguacate (Bower y Cutting 1988; Silveira 2007; Goulao y Oliveira 2008).

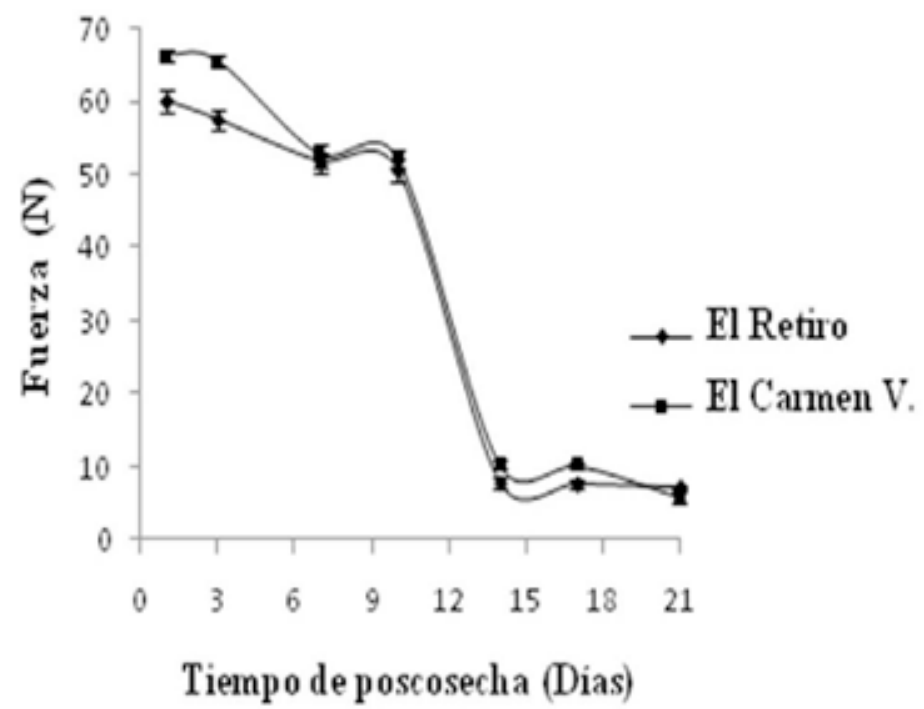

Figura 3. Firmeza de frutos de aguacate (Persea americana Mill. cv. Hass) durante el periodo de poscosecha. Los símbolos representan la media y las barras verticales los valores \pm del error estándar, evaluados durante 21 días de poscosecha. 
Otros estudios han reportado que la pérdida de firmeza es causada por la acción de la pectinmetilesterasa (PME) que elimina grupos de metilo de ácido galacturónico esterificado, lo que aumenta con la maduración de la fruta y mejora la accesibilidad de la poligalacturonasa (PG) al sustrato péctico aumentando la actividad $\beta$-galactosidasa durante las últimas etapas de maduración (Karakurt y Huber 2003).

\section{Rendimiento en mesocarpio (pulpa), epidermis y semilla de los frutos de aguacate}

La tabla 1 presenta los resultados de las fracciones encontradas de los frutos de aguacate para cada día de poscosecha, el rendimiento en pulpa para el día 14 (fruto maduro) fue de $69,8 \%$ y $64,8 \%$ para los municipios de El Carmen de Viboral y de El Retiro respectivamente, los cuales fueron superiores a lo establecido por la Norma Técnica Colombiana (NTC, 5209) de 55,7\% e inferiores a lo determinado por Parra (2005) de $72 \%$. El porcentaje promedio de semilla fue de $15,1 \%$ y $16,8 \%$ para los municipios de El Carmen de Viboral y de El Retiro respectivamente. El porcentaje promedio de la cáscara o epidermis fue de 18,9\% y 21,5\% para los municipios de El Carmen de Viboral y de El Retiro. Los porcentajes de mesocarpio (pulpa), epidermis (cáscara) y semilla, fueron similares a los determinados en 13 variedades de aguacate cultivadas en Venezuela, cuyos valores para pulpa estuvieron entre $55,7 \%$ y $76,8 \%$; para semilla entre $11,8 \%$ y $27,5 \%$ y superiores para la cáscara, que estuvo entre el 7,3\% y 12,7\% (Gómez-López 2002). Para 16 variedades cultivadas en Brasil, se reportaron porcentajes de pulpa entre $60,3 \%$ y $81,1 \%$, de semilla entre $10,0 \%$ y $29,6 \%$ y de cáscara entre $5,1 \%$ y $21,6 \%$ (Tango et al. 1969).

\section{Contenido de humedad y materia seca de los frutos de aguacate}

De acuerdo a la tabla 2 los frutos de aguacate de ambos municipios durante la poscosecha presentaron una disminución continua en la humedad y una relación inversa con la acumulación de materia seca. Durante ésta etapa de poscosecha, los aguacates tuvieron una pérdida total de humedad de $9,7 \%$ y $8,4 \%$ para los municipios de El Carmen de Viboral y de El Retiro.

Tabla 1. Participación de las fracciones de frutos de aguacate (Persea americana Mill. cv. "Hass") durante el periodo de almacenamiento, correspondiente a 21 días de poscosecha.

\begin{tabular}{ccccccc}
\hline $\begin{array}{c}\text { Día } \\
\text { Poscosecha }\end{array}$ & $\begin{array}{c}\text { Mesocarpio } \\
(\mathbf{\%}) \\
\text { El Retiro }\end{array}$ & $\begin{array}{c}\text { Mesocarpio } \\
\mathbf{( \% )} \\
\text { El Carmen }\end{array}$ & $\begin{array}{c}\text { Epidermis } \\
(\mathbf{\%}) \\
\text { El Retiro }\end{array}$ & $\begin{array}{c}\text { Epidermis } \\
(\%) \\
\text { El Carmen }\end{array}$ & $\begin{array}{c}\text { Semilla } \\
(\%) \\
\text { El Retiro }\end{array}$ & $\begin{array}{c}\text { Semilla } \\
(\%) \\
\text { El Carmen }\end{array}$ \\
\hline 1 & $60,6 \pm 0,4$ & $63,6 \pm 0,9$ & $23,1 \pm 0,4$ & $19,8 \pm 0,73$ & $16,2 \pm 0,3$ & $16,6 \pm 0,4$ \\
3 & $63,0 \pm 0,5$ & $65,8 \pm 0,7$ & $21,1 \pm 0,6$ & $19,4 \pm 0,82$ & $15,9 \pm 1,1$ & $15,2 \pm 0,9$ \\
7 & $62,1 \pm 0,3$ & $65,4 \pm 0,9$ & $24,9 \pm 0,5$ & $20,4 \pm 0,85$ & $15,1 \pm 0,6$ & $14,2 \pm 0,4$ \\
10 & $60,1 \pm 1,4$ & $65,0 \pm 0,8$ & $20,7 \pm 0,8$ & $18,1 \pm 0,79$ & $17,8 \pm 0,7$ & $16,0 \pm 1,3$ \\
14 & $64,8 \pm 0,6$ & $69,8 \pm 1,2$ & $15,9 \pm 1,1$ & $15,8 \pm 0,51$ & $17,8 \pm 0,6$ & $15,1 \pm 1,1$ \\
17 & $64,3 \pm 0,5$ & $67,3 \pm 1,1$ & $17,9 \pm 0,9$ & $16,4 \pm 1,09$ & $17,8 \pm 0,6$ & $15,4 \pm 0,7$ \\
21 & $57,4 \pm 0,9$ & $64,5 \pm 1,0$ & $26,9 \pm 0,9$ & $22,2 \pm 1,40$ & $17,1 \pm 0,4$ & $15,5 \pm 1,0$ \\
\end{tabular}

Los valores muestran la media y el error estándar 
La disminución en la humedad es mayor a la encontrada en cv. "Hass" almacenados por 8 días a $15^{\circ} \mathrm{C}$ cuyo valor fue $4,9 \%$ (Villa et al. 2010).

Para el día 10 de poscosecha la materia seca aumentó de 29,3\% a 40,2\% en los frutos de $\mathrm{El}$ Carmen de Viboral y de $28,7 \%$ a $39,4 \%$ en los frutos de El Retiro. Estos valores son mayores a los reportados por Requejo (1999) quien encontró un incremento de $24,6 \%$ a $35,2 \%$ en aguacate cv. "Hass". Parra (2005) reportó para frutos de aguacate valores de materia seca entre $17,4 \%$ a $35,5 \%$. Villa et al. (2010) encontraron valores de $31,6 \%$ a $36,5 \%$ para el día 0 y 8 de poscosecha respectivamente. El incremento en el contenido de aceite en el fruto de aguacate tiene una estrecha relación con la disminución en el porcentaje de agua durante la maduración (Lee 1981).

Lo anterior coincide también con lo señalado por Esteban (1993) respecto a que el contenido de aceite aumenta cuando crece el porcentaje de materia seca, mientras que el contenido de humedad disminuye. Esta relación inversa se atribuye a un desplazamiento de las moléculas de agua por parte de las inclusiones oleosas fuera de las vacuolas celulares, contribuyendo así a reducir el contenido de humedad (Davenport y Ellis 1959).

Entre los días 14 a 21 de la etapa poscosecha, los frutos de aguacate presentaron una disminución en el contenido de materia seca, lo cual coincide con lo reportado por Villa et al. (2010), quienes hallaron una disminución de $36,5 \%$ a $32,9 \%$ entre los días 8 a 12 de poscosecha.

El porcentaje de materia seca se ha adoptado en países como Mexíco y Chile como un importante índice de madurez para aguacate, dado que utiliza técnicas analíticas simples y rápidas, comparado con la determinación del contenido de lípidos (Gaydouy Ratovohery 1987; Kaiser y Wolstenholme 1994; Kruger et al. 1995; Kaiser et al. 1996; Lu et al. 2009).

Tabla 2. Efecto de los días poscosecha sobre la humedad y materia seca de frutos de aguacate (Persea americana Mill. cv. "Hass") almacenados durante 21 días de poscosecha

\begin{tabular}{ccccc}
\hline $\begin{array}{c}\text { Día } \\
\text { Poscosecha }\end{array}$ & $\begin{array}{c}\text { Humedad } \\
(\%) \\
\text { El Retiro }\end{array}$ & $\begin{array}{c}\text { Humedad } \\
(\mathbf{\%}) \\
\text { El Carmen }\end{array}$ & $\begin{array}{c}\text { Materia seca } \\
(\%) \\
\text { El Retiro }\end{array}$ & $\begin{array}{c}\text { Materia seca } \\
(\%) \\
\text { El Carmen }\end{array}$ \\
\hline 1 & $71,3 \pm 0,4$ & $70,6 \pm 0,4$ & $28,66 \pm 0,45$ & $29,3 \pm 0,4$ \\
3 & $68,3 \pm 0,9$ & $68,2 \pm 0,5$ & $31,68 \pm 0,91$ & $31,7 \pm 0,6$ \\
7 & $66,8 \pm 0,7$ & $65,9 \pm 0,9$ & $33,16 \pm 0,73$ & $34,0 \pm 0,9$ \\
10 & $60,6 \pm 0,5$ & $59,8 \pm 0,3$ & $39,35 \pm 0,52$ & $40,2 \pm 0,4$ \\
14 & $62,9 \pm 0,5$ & $60,3 \pm 0,1$ & $37,01 \pm 0,58$ & $39,7 \pm 0,2$ \\
17 & $62,9 \pm 0,1$ & $60,3 \pm 0,4$ & $37,01 \pm 0,11$ & $39,6 \pm 0,4$ \\
21 & $62,9 \pm 0,7$ & $60,9 \pm 0,2$ & $37,01 \pm 0,71$ & $39,0 \pm 0,2$ \\
\hline
\end{tabular}

Los valores muestran la media y el error estándar 


\section{Sólidos solubles totales (SST)}

En la figura 4 puede observarse el incremento de la concentración de SST desde el día 1 hasta el día 14 de poscosecha, alcanzando una concentración de $2,45^{\circ}$ Brix y $2,0^{\circ}$ Brix en los frutos de los municipios de El Carmen de Viboral y de El Retiro. Período en el cual la fruta ha mostrado el pico máximo de producción de $\mathrm{CO}_{2}$, aspecto que probablemente es debido a la actividad metabólica que involucra el paquete enzimático de las $\alpha$ y $\beta$ amilasas, las cuales durante la maduración, hidrolizan el almidón a carbohidratos más simples del tipo disacáridos y monosacáridos (DeMan 1999; Kader 2002).

Otros investigadores han reportado SST de $4,7^{\circ}$ Brix para frutos de aguacate (Persea americana Mill. cv. Fuerte) en estado de madurez de consumo (Schwartz et al. 2007).

\section{Acidez total y pH}

En la tabla 3 se observa como los frutos de aguacate de ambos municipios presentaron una disminución en la acidez.
Entre los días 1 a 10 de poscosecha se mostró un aceleramiento en la pérdida de acidez, disminuyendo de $0,25 \%$ a $0,18 \%$ y de $0,24 \%$ a $0,16 \%$ expresada como ácido oleico para los frutos del municipio de El Carmen de Viboral y de El Retiro. El pH presentó un comportamiento inverso, aumentando de 6,42 a 6,63 Unidades de $\mathrm{pH}$ para los frutos de El Carmen de Viboral y de 6,43 a 6,54 para los frutos de El Retiro. Los valores encontrados para el $\mathrm{pH}$ concuerdan con lo reportado para frutos de aguacate cv. Hass en estado de madurez de consumo (Hess et al. 1993; Camarena et al. 2000).

De acuerdo al análisis de correlación para materia seca y contenido de humedad, se pudo establecer que existe una alta correlación, correspondiente al 0,99 para ambos municipios, la cual se ajusta a un modelo potencial de grado dos.

Se pudo establecer para ambos municipios como a medida que existe una mayor concentración de materia seca esta se correlaciona de manera inversa con el

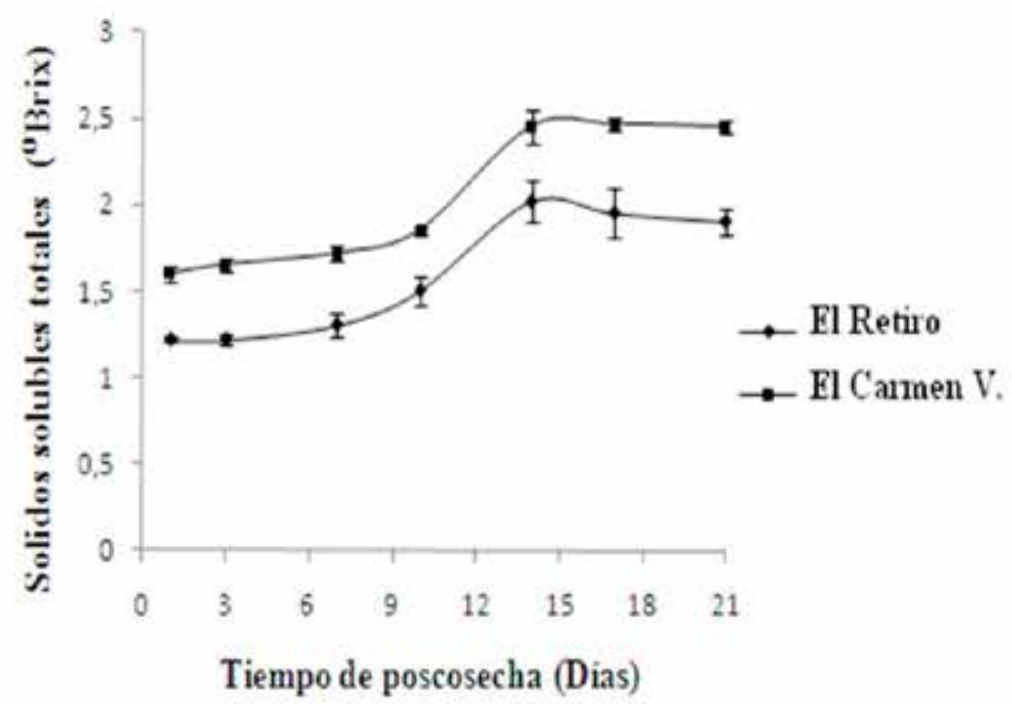

Figura 4. Evolución de los sólidos solubles totales para frutos de aguacate (Persea americana Mill. cv. "Hass") de dos municipios de Antioquia Colombia. Los símbolos representan la media y las barras verticales los valores \pm del error estándar, evaluados durante 21 días de poscosecha. 
Tabla 3. Efecto de los días poscosecha sobre el $\mathrm{pH}$ y la acidez de frutos de aguacate durante el periodo de almacenamiento

\begin{tabular}{ccccc}
\hline $\begin{array}{c}\text { Día } \\
\text { Poscosecha }\end{array}$ & $\begin{array}{c}\mathbf{p H} \\
\text { El Retiro }\end{array}$ & $\begin{array}{c}\mathbf{p H} \\
\text { El Carmen V. }\end{array}$ & $\begin{array}{c}\text { Acidez } \\
\text { \%ác. Oleíco) } \\
\text { El Retiro }\end{array}$ & $\begin{array}{c}\text { Acidez } \\
\text { (\% ác. Oleíco) } \\
\text { El Carmen V. }\end{array}$ \\
\hline 1 & $6,43 \pm 0,002$ & $6,42 \pm 0,003$ & $0,24 \pm 0,01$ & $0,25 \pm 0,01$ \\
3 & $6,45 \pm 0,007$ & $6,44 \pm 0,001$ & $0,24 \pm 0,01$ & $0,24 \pm 0,01$ \\
7 & $6,53 \pm 0,003$ & $6,60 \pm 0,002$ & $0,18 \pm 0,01$ & $0,21 \pm 0,01$ \\
10 & $6,54 \pm 0,004$ & $6,63 \pm 0,000$ & $0,17 \pm 0,01$ & $0,19 \pm 0,00$ \\
14 & $6,57 \pm 0,007$ & $6,63 \pm 0,008$ & $0,16 \pm 0,01$ & $0,17 \pm 0,01$ \\
17 & $6,57 \pm 0,001$ & $6,63 \pm 0,001$ & $0,16 \pm 0,00$ & $0,16 \pm 0,01$ \\
21 & $6,57 \pm 0,002$ & $6,63 \pm 0,003$ & $0,16 \pm 0,013$ & $0,16 \pm 0,01$ \\
\hline
\end{tabular}

Los valores muestran la media y el error estándar

contenido de humedad, lo cual esta a su vez relacionado con la concentración lipídica del vegetal.

La disminución de la acidez durante la etapa poscosecha puede ser debida probablemente al consumo de moléculas orgánicas en los diferentes ciclos metabólicos para proporcionar la energía requerida por el fruto, además muchos de los ácidos orgánicos pueden participar como precursores de sustancias volátiles, las cuales intensifican su presencia durante este período (Park et al. 2006).

\section{Contenido lipídico de la pulpa de aguacate}

De acuerdo a la figura 5 el contenido de aceite es superior para los frutos de aguacate provenientes del municipio de El Carmen de Viboral durante toda la etapa de poscosecha. Para la madurez de consumo correspondiente al día 14 se encontró un contenido de aceite del $23,8 \%$ y $22,4 \%$ para los frutos de El Carmen de Viboral y El Retiro. En el período de sobremadurez correspondiente a los días 17 a 21 de poscosecha no se presentaron cambios significativos en la concentración de aceite.
Los valores encontrados en el contenido de aceite fueron superiores a los reportados por Olaeta et al. (1999), quienes hallaron concentraciones de aceite entre el $12,9 \%$ y $14,6 \%$ para aguacate cv. "Hass". Mientras que otros investigadores hallaron en frutos de aguacate en madurez de consumo concentraciones de aceite del 19,9\% (Villa et al. 2010). Otra investigación reporta una concentración de aceite del 22,5\% para aguacate cv. Hass en madurez de consumo (Woolf et al. 2009).

\section{CONCLUSIONES}

La tasa de respiración de los frutos de aguacate los tipifica como climatéricos monofásicos, presentando el pico climatérico en el día 8 de poscosecha con una producción de $152 \mathrm{mg}$ de $\mathrm{CO}_{2} \bullet \mathrm{kg}^{-1} \bullet \mathrm{h}^{-1}$.

La pérdida fisiológica de peso y la firmeza, presentaron un comportamiento decreciente durante toda la etapa de maduración de los frutos de aguacate, para los dos municipios estudiados. 


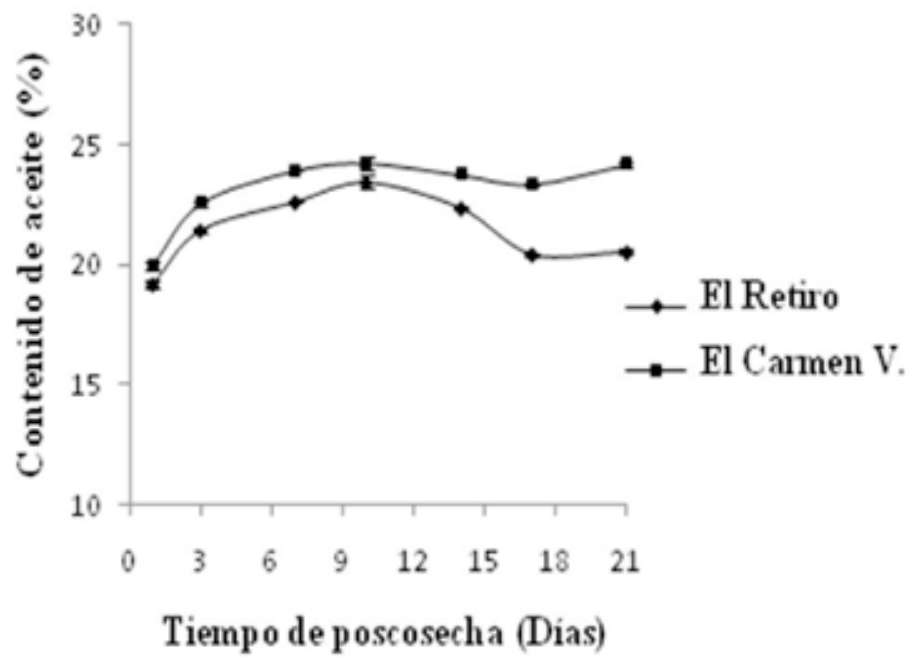

Figura 5. Contenido de aceite en frutos de aguacate (Persea americana Mill. cv. "Hass"). Los símbolos representan la media y las barras verticales los valores \pm del error estándar, evaluados durante 21 días de poscosecha.

Las coordenadas de color $L^{*}, a^{*}$ y $b^{*}$, especialmente la luminosidad permitió establecer un intervalo de valores que se puede asociar a la madurez óptima del fruto, lo cual fue común para todos los frutos estudiados.

La mayor concentración de aceite, materia seca y SST en los frutos de aguacate, se presentó entre los días 12 y 14 de poscosecha, etapa que coincidió con la madurez de consumo del vegetal.

\section{REFERENCIAS}

Agustí, M. 2004. Fruticultura. Ediciones Mundi-Prensa. España. 163 p.

Almela, L. 2000. Non destructive appraisal of the ripennness in cantaloupe melons. Food Science Technology. 6: 47-51.

Angueira, M., Sandoval, A. y Barreiro, J. 2003. Tasas de respiración en cuatro híbridos de pimentón (Capsicum annum L.). Interciencia. 28: 1-9.
Association Official Analytical Chemist. (A.O.A.C.). 1984. Official methods on analysis of Association of Official Analytical Chemist. 14thed. Richmond. Virginia, U.S.A. 1. 141 p.

Award, M. and Young, R. 1979. Postharvest variation in cellulase, polygalacturonase and pectinmethylesterase in avocado (Persea americana Mill Cv. Fuerte) fruits in relation to respiration and ethylene production. Plant Physiol. 64: 306-308.

Bernal, I. 1993. Análisis de Alimentos. Universidad Nacional de Colombia, Santafé de Bogotá. 127 p.

Bower, J. and Cutting, J. 1988. Avocado fruit development and ripening physiology. Hort. Rev. 10: 229-271.

Bruinsma, J. and Paull, P. 1984. Respiration during postharvest development of soursop fruit (Annona muricata L.). PlantPhysiol. 76(1): 131-138. 
Cajuste, B., Saucedo, V. y Colinas L. 1994. Comportamiento poscosecha de fruto de aguacate (cv Hass) en función de la época de corte. Revista Fitociencia Mexicana.17(1): 94-102.

Camacho, G. y Romero, G. 1996. Obtención y conservación de pulpas: Mora, guanábana, lulo y mango. Bogotá: ICTA - Universidad Nacional de Colombia, $130 \mathrm{p}$.

Camarena, B. 2000. Caracterización sensorial y fisicoquímica de la pulpa de aguacate Variedad Hass. Instituto Tecnológico de Celaya; Antonio García Cubas. IX Congreso de Ciencia de los Alimentos y $\mathrm{V}$ Foro de Ciencia y Tecnología de Alimentos. Instituto de Ciencias Agrícolas, Universidad de Guanajuato. México.

Correa, P., Plaza, J. y Ruiz, M. 1995. Ensayos no destructivos para la evaluación de la madurez post-recolección de aguacate. Agro-Ciencia. 11(2): 197-200.

Cox, K. 2004. Skin colour and pigment changes during ripening of 'Hass' avocado fruit. Postharvest Biology and Technology. 31: 287-294.

Davenport, J. and Ellis, S. 1959. Chemical changes during growth and storage of the avocado fruit. Austral. J. Biol. Sci. 12: 445-454.

Deman, M.1999. Principles of food chemistry. Third Edition. Guelph, Ontario: Aspen Publishers, Inc. 595 p.
Devia, J. 2009. Innovación para el desarrollo de productos. Grupo de investigación desarrollo y diseño de procesos y productos, Departamento de Ingeniería de Procesos. Universidad EAFIT. No 122. Medellín, 13 p.

Eaks, I. 1978. Ripening, respiration and ethylene production of 'Hass' avocado fruits at $20^{\circ}$ to $40^{\circ} \mathrm{C}$. C. J. Amer. Soc. Hort. Sci. 103: 576-578.

Esteban, P. 1993. Estimación del contenido de aceite, a través de la humedad y su relación con palatabilidad en frutos de palto de las variedades Negra de la Cruz, Bacon, Edranol y Hass, desde la última etapa de desarrollo hasta la madurez fisiológica. Tesis Ing. Agr. Quillota. U. Católica de Valparaíso. Escuela de Agronomía. 54 p.

\section{Gaydou, E., Lozano, Y. and Ratovohery,} J. 1987. Triglyceride and fatty acid compositions in the mesocarp of Persea americana during fruit development. Phytochemistry. 26: 1595-1597.

Gómez, V. 2002. Fruit characterization of high oil content avocado varieties. Sci. agric. 55(2): 403-406.

Goulao, L. and Oliveira, C. 2008. Cell wall modifications during fruit ripening. When a fruit is not the fruit. Trends in Food Science and Technology. 19(1): 4-25.

Gutiérrez, C. 2009. Análisis del mercado para aguacate. El Salvador. 6 p. 
Herridge, F. and Pate, S. 1977. Utilization of net photosynthate for nitrogen fixation and protein production in an annual legume.Plant Physiol. 60: 759-764.

Hess, B. and Kader, A. 1993. Changes in intracellular $\mathrm{pH}, \mathrm{ATP}$, and glycolytic enzymes in 'Hass' avocado in response to low $\mathrm{O}_{2}$ and high $\mathrm{CO}_{2}$ stresses. Proc. 6th Intl. Controlled Atmosphere Res. Conf., Cornell U., Ithaca, N.Y. 9 p.

Islam, S., Matsui, T. and Yoshida, Y. 1996. Effect of carbon dioxide enrichment on physico-chemical and enzymatic changes in tomato fruits at various stages of maturity. Scientia Horticulturae. 65(23): 137-149.

Kader, A. 2005. Increasing food availability by reducing potharvest losses of fresh produce. Acta Hort. 682: 2169-2175.

Kader, A. 1991. Índices de madurez, factores de calidad, normalización e inspección de productos hortícolas. en: Yahía, E.; Higuera, C. (eds.), Fisiología y tecnología postcosecha de productos hortícolas. Centro de Investigación en Alimentación y Desarrollo. México D.F. México. p49-58.

Kader, A. 2002. Postharvest technology of horticultural crops. Agriculture and Natural Resources. Davis, California: University of California. 535 p.

Kaiser, C. and Wolstenholme, B. 1994. Aspects of Delayed Harvest of Hass Avocado (Persea Americana Mill.) Fruit in Cool Subtropical Climate. I. Fruit lipid and fatty acid accumulation. Journal of Horticultural Science. 69(3): 437-445.

Kaiser, C., Levin, J. and Wolstenholme, B. 1996. Towards Maturity Standards for Fuerte Avocado Fruit in the Cool Subtropical Kwazulu-Natal Midlands.South African Avocado.Growers Association Yearbook. 19: 101-108.

Karakurt, Y. and Huber, D. 2003. Activities of several membrane and cell-wall hydrolases, ethylene biosynthetic enzymes, and cell wall polyuronide degradation during low-temperature storage of intact and fresh-cut papaya (Carica papaya) fruit. Postharvest Biology and Technology. 28(2): 219-229.

Kruger, F, Stassen, P. and Snijders, B. 1995. Preliminary Study on Variation in the Maturity Parameters of Avocados from the Kiepersol- Hazyviewarea. South African Avocado. Growers Association Yearbook. 5: 74-77.

Lallum, N. 2004. Role of water loss in ripening of 'Hass' avocados. New Zealand Avocado Growers Association Annual Research Report. 4: 70-79.

Lee, S. 1981. Review and backround of avocado maturity standard. California Avocado Soc. Yearbook. 65: 101-109.

Lu, Q. 2009. California Hass avocado: profiling of carotenoids, tocopherols, fatty acids, and fat content during maturation and from different growing areas. J.Agric Food Chem. 57 (21): 10408-10413. 
Manenoi, A. 2007. Utility of 1-methylcyclopropene as a papaya postharvest treatment. Postharvest Biology and Technology. 44: 55-62.

Márquez, C. 2009. Caracterización fisiológica, físico-química, reológica, nutraceútica, estructural y sensorial de la guanábana (Annona Muricata L. cv. elita). Universidad Nacional de Colombia sede Medellín, Facultad de Ciencias Agropecuarias, Departamento de Ciencias Agronómicas. Medellín. Tesis Doctoral. 274 p.

\section{Ministerio de Agricultura y Desarrollo Rural.} 2012. Agronet. Bogotá D.C. 20 p.

Moreno Ortiz, A. 2007. Estudio comparativo del efecto hipolipidémico inducido por aceites monoinsaturados de aguacate. Proceedings VI World Avocado Congress (Actas VI Congreso Mundial del Aguacate). Viña del Mar, Chile, 12-16 nov. $11 \mathrm{p}$.

Olaeta, J., Undurraga, P. y Schwartz, M. 1999. Determinación de la evolución y caracterización de los aceites en paltas (Persea americana Mill.) cvs. Fuerte y Hass cultivados en Chile. Revista Chapingo, Serie Horticultura. 5: 117-122.

Park, Y., Jung, S. and Gorinstein, S. 2006. Ethylene treatment of 'Hayward' kiwifruits (Actinidia deliciosa) during ripening and its influence on ethylene biosynthesis and antioxidant activity. ScientiaHorticulturae. 108: 22-28.
Parra, C., Hernández, H. 2005. Fisiología postcosecha de frutas y hortalizas. Bogotá: Universidad Nacional de Colombia. 66 p.

Ramírez, J. 2008. Boletín informativo, Central Mayorista de Antioquia, Medellín. 25 p.

Requejo, C. 1999. International trends in fresh avocado and avocado oil production and seasonal variation of fatty acids in New Zealand-grown cv. Hass. University New Zealand. Tesis de Maestría. 258 p.

Silveira, A. 2007. Fisiología y bioquímica de los productos MPF, V Congreso Iberoamericano de Tecnología Postcosecha y Agroexportaciones. Cartagena-España: Universidad de Cartagena. Ed. Universidad Politécnica de Cartagena. 1655 p.

Tango, J. 1969. Composiçâo do fruto e do óleo de diferentes variedades de abacate cultivadas no estado de Sâo Paulo. Coletânea do Instituto de Tecnología de Alimentos. 3: 283-292.

Tejacal, I. 2007. Postharvestphysiology and technology of sapotemamey fruit (Pouteria sapota (Jacq.) H.E. Moore \&Stearn). Review Postharvest Biology and Technology. 45(3): 285-297.

Velázquez, J. 2008. Identificación del aguacate como un rubro importante de grandes oportunidades comerciales, según los acuerdos de integración, los nuevos tratados comerciales y el comercio mundial globalizado. Secretaría de Productividad 
y Competitividad, Gobernación de Antioquia. Colombia, 103 p.

Villa R., Molina C., Ayala Z., Guadalupe I. e González, A. 2010. Effect of maturity stage on the content of fatty acids and antioxidant activity of "Hass" avocado. Food Research International. 44: 12311237.

Woolf, A. 2009. Avocado Oil.Reprinted with permission from Gourmet and HealthPromoting Specialty Oils Edited by Robert A. Moreau and Afaf Kamal-Eldin, AOCS Press, Urbana, Illinois. Copyright ( AOCS Press. p541-550.
Wu, B. 2005. Changes in sugar and organic acid concentrations during fruit maturation in peaches, (Prunus davidiana) and hybrids as analyzed by principal component analysis. ScientiaHorticulturae. 103(4): 429-439.

Yang, X. 2009. Characterization of chlorophyll degradation in banana and plantain during ripening at high temperature. Food Chemistry. 114(2): 383-390.

Zauberman, G. and Jobin, M. 1995. Avocado (Persea americana Mill.) quality changes in response to low temperature storage. Postharvest Biology and Technology. 5: 235-243.

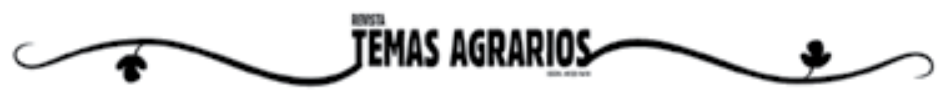

